\title{
In congenital hypothyroidism bone maturation at birth may be a predictive factor of psychomotor development during the first year of life irrespective of other variables related to treatment
}

\author{
Malgorzata Wasniewska, Filippo De Luca, Alessandra Cassio ${ }^{1}$, Nicola Oggiaro $^{2}$, Paola Gianino ${ }^{3}$, \\ Maurizio Delvecchio ${ }^{4}$, Rosalba Aiazzi ${ }^{1}$, Vera Stoppioni ${ }^{2}$, Fortunato Lombardo, Maria Francesca Messina, \\ Mariella Valenzise and Teresa Arrigo \\ Departments of Pediatrics, Universities of Messina, ${ }^{1}$ Bologna, ${ }^{2}$ Ancona, ${ }^{3}$ Torino and ${ }^{4}$ Bari, Italy \\ (Correspondence should be addressed to F De Luca, Dipartimento di Scienze Pediatriche Mediche e Chirurgiche, Policlinico Universitario di Messina, \\ Via Consolare Valeria, 98123 Messina, Italy; Email: wasniewska@yahoo.it)
}

\begin{abstract}
Objective: To evaluate in a cohort of infants with congenital hypothyroidism (CH): (a) the frequency of bone maturation $(\mathrm{BM})$ retardation at birth and (b) whether BM delay at birth may be considered as a tool to make a prognosis of psychomotor status at the age of 1 year, irrespective of other variables related to treatment.

Design: BM at birth, $\mathrm{CH}$ severity and developmental quotient (DQ) at the age of 1 year were retrospectively evaluated in $192 \mathrm{CH}$ infants selected by the following inclusion criteria: (a) gestation age ranging between 38 and 42 weeks; (b) onset of therapy within the first month of life; (c) initial thyroxine (L- $\mathrm{T}_{4}$ ) dosage ranging from 10 to $12 \mu \mathrm{g} / \mathrm{kg} /$ day; (d) normalization of serum thyrotropin (TSH) levels before the age of 3 months; (e) monthly adjustments of $\mathrm{L}_{-} \mathrm{T}_{4}$ dose during the first year of life with serum TSH levels ranging from 0.5 to $4 \mathrm{mIU} / \mathrm{l}$; (f) no major diseases and/or physical handicaps associated with $\mathrm{CH}$; $(\mathrm{g})$ availability of both thyroid scanning and knee X-rays at the time of treatment initiation; (h) availability of DQ assessment at an average age of 12 months.

Methods: BM was considered normal if the distal femur bony nucleus diameter exceeded $3 \mathrm{~mm}$ (group A) or retarded if either this nucleus was absent (subgroup B1) or its diameter was $<3 \mathrm{~mm}$ (subgroup B2). DQ was evaluated with the Brunet-Lézine test.

Results: In $44.3 \%$ of cases BM was either delayed (23.5\%) or severely delayed (20.8\%). The risk of BM retardation was higher in the patients with athyreosis than in the remaining patients $(41 / 57 \mathrm{vs}$ 44/135, $\left.\chi^{2}=25.13, P<0.005\right)$. BM-retarded infants showed a more severe biochemical picture of $\mathrm{CH}$ at birth and a lower $\mathrm{DQ}$ at the age of one year compared with the group A patients. If compared with infants of subgroup B2 those of subgroup B1 exhibited significantly lower $\mathrm{T}_{4}$ levels at birth and a more frequent association with athyreosis $\left(70.0\right.$ vs $\left.30.0 \% ; \chi^{2}=7.49, P<0.01\right)$, whereas DQ was superimposable in both subgroups.

Conclusions: (a) BM at birth is delayed in almost half of $\mathrm{CH}$ patients and (b) $\mathrm{CH}$ severity per se can affect $\mathrm{DQ}$ at the age of 1 year irrespective of other variables related to therapy.
\end{abstract}

European Journal of Endocrinology 149 1-6

\section{Introduction}

Neonatal screening programs have led to improved intellectual prognosis for children with congenital hypothyroidism $(\mathrm{CH})$, thanks to the significant anticipation of the start of replacement therapy. Nevertheless, subtle neurocognitive deficits may be detected even in $\mathrm{CH}$ children treated very early in life $(1,2)$, which confirms that the timing of onset of treatment is not the only factor involved in the regulation of psychomotor development in $\mathrm{CH}$ infants. Another important factor that is able to influence intellectual prognosis in these patients is the severity of $\mathrm{CH}$ at diagnosis, as assessed by serum thyroxine $\left(\mathrm{T}_{4}\right)$ levels, amount of thyroid tissue and the degree of bone maturation (BM) $(3-8)$. $\mathrm{BM}$ retardation at birth has been proposed as an indicator of intrauterine and severe $\mathrm{CH}$, with a negative prognostic value on long-term intellectual outcome (9-12). Nonetheless, its prevalence among the neonates with $\mathrm{CH}$ has not been investigated hitherto.

The aims of the present study were to evaluate: the frequency of $\mathrm{BM}$ retardation at birth in our $\mathrm{CH}$ population; whether BM retardation at birth is associated with other biological signs of intrauterine and severe 
$\mathrm{CH}$; whether BM delay at birth may be considered as a tool to make a prognosis of psychomotor status at the age of one year, irrespective of other variables related to treatment; and whether a different degree of BM delay at birth correlates with the severity of $\mathrm{CH}$ and may be associated with a different psychomotor status at the age of 1 year.

\section{Patients and methods}

\section{Study population}

This retrospective study covers a cohort of 192 infants with persistent $\mathrm{CH}$ who were selected from a larger population of $\mathrm{CH}$ patients identified by regional screening programs during a 10-year period between 1990 and 1999.

Screening programs were based on thyrotropin (TSH) and $\mathrm{T}_{4}$ analyses of filter paper blood samples collected on the fifth day of life. The cut-off TSH value used to discriminate newborns with $\mathrm{CH}$ from unaffected infants was $20 \mathrm{mIU} / \mathrm{l}$, whereas the concentration of the highest standard of the spot TSH assay was $400 \mathrm{mIU} / \mathrm{l}$. In all the patients, diagnosis of $\mathrm{CH}$ had been confirmed at recall on the basis of abnormal serum TSH, total $\mathrm{T}_{4}$ and free $\mathrm{T}_{4}\left(\mathrm{FT}_{4}\right)$ measurements.

The 192 subjects admitted to our study were selected by the following inclusion criteria: gestational age ranging between 38 and 42 weeks; onset of therapy by the 1st month of life (mean 23 \pm 2 days; range 14-30); initial $\mathrm{L}^{-} \mathrm{T}_{4}$ dosage ranging from 10 to $12 \mu \mathrm{g} / \mathrm{kg} /$ day; normalization of TSH serum levels $(<4 \mathrm{mIU} / \mathrm{l})$ before the age of 3 months; monthly evaluations of TSH and total $\mathrm{T}_{4}$ serum levels and adjustments of the $\mathrm{L}-\mathrm{T}_{4}$ dose during the 1st year of life, in order to maintain a euthyroid status, with TSH serum levels ranging from 0.5 to $4.0 \mathrm{mIU} / \mathrm{l}$; no major diseases and/or physical handicaps associated with $\mathrm{CH}$; availability of both thyroid scanning and knee X-rays, which had already been performed at the time of initiation of treatment in order to assess either etiological diagnosis of $\mathrm{CH}$ or neonatal skeletal maturity respectively; availability of a developmental quotient (DQ) assessment at an average age of 12 months.

\section{Methods}

Thyroid scanning at confirmation of diagnosis had been performed by either $99 \mathrm{~m}$ Technetium or 123 Iodine and the 192 patients selected for this study were classified as having athyreosis $(57 / 192=29.7 \%)$ or other types including ectopic thyroid gland $(96 / 192=50 \%)$ and normally sited gland $(39 / 192=20.3 \%)$.

Skeletal maturation was evaluated by X-ray assessment of the distal femoral epiphyseal ossification center. In order to minimize the influence of $\mathrm{L}-\mathrm{T}_{4}$ treatment and postnatal factors on skeletal maturation only infants in whom X-rays were obtained within the first month of life were included. Moreover, in all of the selected patients radiography had been performed either before or shortly after the start of therapy (range 1-3 days). In all the infants the presence or absence of the distal femoral epiphyseal nucleus was scored according to the method by Ilicki et al. (13), simplified as follows: bony nucleus diameter was measured on the film using electronic callipers and BM was considered (a) normal if its diameter exceeded $3 \mathrm{~mm}$ (group A) or (b) retarded if this bony nucleus was absent or its diameter was $<3 \mathrm{~mm}$ (group B). This limit was arbitrarily chosen according to the criteria proposed by Sénécal et al. (14) and partially modified by Ilicki et al. (13). The same limit had previously been adopted by Heyerdahl et al. (15). A knee epiphysis diameter $<3 \mathrm{~mm}$ implies that the epiphyseal area is $<0.07 \mathrm{~mm}^{2}$, which is very similar to the cut-off value (epiphyseal area $<0.05 \mathrm{~mm}^{2}$ ) used by Glorieux et al. (10). The patients with retarded BM were further divided into two subgroups: those with absent distal femoral nucleus (subgroup B1) and those with a small nucleus, i.e. $<3 \mathrm{~mm}$ (subgroup B2).

Psychomotor development had been assessed in all the patients with the Brunet-Lézine test. This test had been carried out by different psychologists at the local pediatric clinics when the patients were 10-14 months old (mean 12 \pm 1 months). The BrunetLézine psychomotor developmental scales provide an estimate of overall development considering four specific intellectual and behavioral functions: locomotor and postural control, verbal level, personalsocial relations and oculo-motor coordination (16).

According to this test, DQ in a control population of 9-month-old normal infants has very recently been reported to be in the range from 85 to 105 (average $98 \pm 6.4$ ) (17). In the light of these results DQ in our study population was arbitrarily considered as abnormal if it was lower than 85 .

\section{Statistical analyses}

For statistical purposes, Student's unpaired t-test, Mann-Whitney's test or chi-square test were used when appropriate in order to estimate differences between groups. Correlations on individual values were made by Pearson's test. All values are given as means \pm standard deviation (S.D.).

This study was approved by the Ethical Committees of all the Hospitals participating in this investigation.

\section{Results}

\section{Frequency of BM retardation at birth}

According to our definition for neonatal BM retardation, BM was normal in 107/192 infants (55.7\%) and retarded in the remaining 85 patients $(44.3 \%)$. Among the 85 patients belonging to group B, 45 
exhibited a very severe delay in BM, as indicated by the absence of the distal femur bony nucleus (subgroup $\mathrm{B} 1=23.5 \%)$. In another 40 infants BM retardation was less severe, being substantiated only by femoral bony nucleus hypoplasia (subgroup B2 $=20.8 \%$ ).

\section{Association between BM retardation and other biological signs of intrauterine $\mathrm{CH}$}

Among the 85 infants with neonatal BM retardation, forty-one $(48.2 \%)$ were athyreotic. Since the overall prevalence of athyreosis in the entire study population was 57/192 (29.7\%) the risk of BM retardation was significantly higher in the patients with athyreosis than in those with other causes of $\mathrm{CH}$ (41/57 vs 44/135; $\left.x^{2}=25.13, P<0.0005\right)$.

If compared with the patients of group A, BMretarded infants exhibited a more severe biochemical picture of thyroid failure, as clearly demonstrated by the following evidence: (a) TSH blood concentrations were significantly higher in group B patients both at the time of screening and at recall examination (Table 1); (b) $\mathrm{T}_{4}$ blood concentrations, on the other hand, were significantly lower in BM-retarded infants at both assessments (Table 1). Also $\mathrm{FT}_{4}$ serum levels at recall were slightly lower in group $B$ patients than in those with normal BM, although this difference did not achieve statistical relevance (Table 1).

\section{Relationships between the degree of BM delay and severity of $\mathrm{CH}$}

Among the 85 infants of group B a more severe degree of BM retardation was found in those belonging to subgroup B1 than in those belonging to subgroup B2. Among the 45 patients of subgroup B1, 28 (70\%) were athyreotic, which demonstrated the existence of a more frequent association with athyreosis in this subgroup of infants with severely retarded BM than in those of subgroup B2 $\left(28 / 40\right.$ vs $13 / 45 ; \chi^{2}=7.492$, $P<0.01)$.

Table 1 Thyroid function tests and prevalence of athyreosis in 192 congenitally hypothyroid $(\mathrm{CH})$ infants with either normal (group A) or retarded (group B) bone maturation at birth. Values are means \pm S.D.

\begin{tabular}{lccc}
\hline & $\begin{array}{c}\text { Group A } \\
(107=55.7 \%)\end{array}$ & $\begin{array}{c}\text { Group B } \\
(85=44.3 \%)\end{array}$ & $\boldsymbol{P}$ value \\
\hline $\mathrm{TSH}(\mathrm{mlU} / \mathrm{l})^{\star}$ & $179.3 \pm 161.5$ & $271.0 \pm 172.3$ & $<0.0005$ \\
$\mathrm{TSH}(\mathrm{mlU} / \mathrm{l})^{\star *}$ & $244.2 \pm 248.8$ & $437.4 \pm 677.6$ & $<0.005$ \\
$\mathrm{~T}_{4}(\mathrm{nM} / \mathrm{l})^{\star}$ & $61.7 \pm 38.6$ & $39.9 \pm 29.6$ & $<0.005$ \\
$\mathrm{~T}_{4}(\mathrm{nM} / \mathrm{l})^{\star \star}$ & $55.3 \pm 43.7$ & $27.0 \pm 23.2$ & $<0.0005$ \\
$\mathrm{FT}_{4}(\mathrm{pM} / \mathrm{l})^{\star *}$ & $5.5 \pm 5.0$ & $3.0 \pm 2.0$ & $\mathrm{n} . \mathrm{s}$. \\
Athyreosis $(\%)$ & 28.1 & 71.9 & $<0.0005$
\end{tabular}

*Thyrotropin (TSH) and thyroxine $\left(\mathrm{T}_{4}\right)$ filter paper measurement at the time of screening; **TSH, $\mathrm{T}_{4}$ and free $\mathrm{T}_{4}\left(\mathrm{FT}_{4}\right)$ serum measurement at recall. Statistical tests used: TSH, Mann-Whitney test; $\mathrm{T}_{4}$, Student's unpaired $t$-test; athyreosis, $\chi^{2}$-test. n.s., not significant.
Moreover, if compared with the patients of subgroup B2, those with severely retarded BM exhibited a more severe impairment of thyroid function, as shown by the following biochemical findings: (a) $\mathrm{T}_{4}$ blood concentrations were significantly lower in subgroup B1 both at the time of screening and at the recall assessment (Table 2); (b) average TSH blood concentrations were slightly higher at both assessments and $\mathrm{FT}_{4}$ serum levels were slightly lower at recall in subgroup B1 patients, although these differences did not attain a statistical relevance (Table 2).

\section{Variables related to treatment}

Age at therapy onset, initial $\mathrm{L}_{-} \mathrm{T}_{4}$ dose, age at TSH normalization and average TSH and $\mathrm{T}_{4}$ serum levels during the first year of life under $\mathrm{L}_{-} \mathrm{T}_{4}$ treatment are shown in Table 3. None of these variables were significantly different in the group A patients compared with those of group B.

Table 2 Thyroid function tests and prevalence of athyreosis in 85 congenitally hypothyroid $(\mathrm{CH})$ infants with either absent (subgroup B1) or hypoplasic (subgroup B2) distal femur bony nucleus. Values are means \pm S.D.

\begin{tabular}{lccc}
\hline & $\begin{array}{c}\text { Subgroup B1 } \\
(45=52.9 \%)\end{array}$ & $\begin{array}{c}\text { Subgroup B2 } \\
(40=47.1 \%)\end{array}$ & $\boldsymbol{P}$ value \\
\hline $\mathrm{TSH}(\mathrm{mlU} / \mathrm{l})^{\star}$ & $299.5 \pm 161.5$ & $243.6 \pm 180.5$ & n.s. \\
$\mathrm{TSH}(\mathrm{mlU} / \mathrm{l})^{\star \star}$ & $506.2 \pm 408.7$ & $361.2 \pm 232.5$ & n.s. \\
$\mathrm{T}_{4}(\mathrm{nM} / \mathrm{l})^{\star}$ & $21.8 \pm 12.9$ & $50.2 \pm 30.9$ & $<0.005$ \\
$\mathrm{~T}_{4}(\mathrm{nM} / \mathrm{l})^{\star *}$ & $21.8 \pm 16.7$ & $33.5 \pm 25.7$ & $<0.05$ \\
$\mathrm{FT}_{4}(\mathrm{pM} / \mathrm{l})^{\star \star}$ & $2.3 \pm 2.3$ & $3.5 \pm 2.5$ & $\mathrm{n} . \mathrm{s}$. \\
Athyreosis $(\%)$ & 70.0 & 30.0 & $<0.01$ \\
\hline
\end{tabular}

*Thyrotropin $(\mathrm{TSH})$ and thyroxine $\left(\mathrm{T}_{4}\right)$ filter paper measurement at the time of screening; ${ }^{\star *} \mathrm{TSH}, \mathrm{T}_{4}$ and free $\mathrm{T}_{4}\left(\mathrm{FT}_{4}\right)$ serum measurement at recall.

Statistical tests used: TSH, Mann-Whitney test; $\mathrm{T}_{4}$, Student's unpaired $t$-test; athyreosis, $\chi^{2}$-test. n.s., not significant.

Table 3 Variables related to treatment and developmental quotient (DQ) at the age of 1 year in 192 congenitally hypothyroid $(\mathrm{CH})$ infants with either normal (group A) or retarded (group B) bone maturation at birth.

\begin{tabular}{|c|c|c|c|}
\hline & $\begin{array}{c}\text { Group A } \\
(107=55.7 \%)\end{array}$ & $\begin{array}{c}\text { Group B } \\
(85=44.3 \%)\end{array}$ & $P$ value \\
\hline Therapy onset (days) & $24.0 \pm 2.0$ & $22.0 \pm 3.0$ & n.s. \\
\hline $\begin{array}{l}\text { Initial } L-T_{4} \text { dosage } \\
(\mu \mathrm{g} / \mathrm{kg} / \text { day })\end{array}$ & $11.1 \pm 3.7$ & $10.8 \pm 2.0$ & n.s. \\
\hline $\begin{array}{l}\text { TSH normalization } \\
\text { (weeks) }\end{array}$ & $4.3 \pm 3.4$ & $2.1 \pm 1.8$ & n.s. \\
\hline Average TSH (mIU/l)* & $1.5 \pm 0.9$ & $1.8 \pm 0.6$ & n.s. \\
\hline Average $\mathrm{T}_{4}$ (nM.I)* & $166.0 \pm 5.1$ & $169.9 \pm 7.7$ & n.s. \\
\hline $\mathrm{DQ}$ at the age of 1 year & $103.9 \pm 11.3$ & $95.1 \pm 9.3$ & $<0.0005$ \\
\hline
\end{tabular}

*Average thyrotropin (TSH) and thyroxine $\left(\mathrm{T}_{4}\right)$ serum levels during the first year of life under $\mathrm{L}-\mathrm{T}_{4}$ therapy.

Statistical tests used: all parameters except average TSH (Mann-Whitney test) were compared using Student's unpaired $t$-test. n.s., not significant. 


\section{Psychomotor development assessment at the age of one year}

In the overall study population average DQ at the age of 1 year was $100.1 \pm 11.3$ (range 80-130), with a significant difference $(P<0.0005)$ between the patients belonging to group A (mean 103.9 \pm 11.3 , range 80-130) and those included in group B (mean 95.1 \pm 9.3 , range 80-113) (Table 3). No differences in terms of $\mathrm{DQ}$ were found between the patients included in subgroups B1 (mean 95.2 \pm 10.2 , range $80-113)$ and B2 (mean 94.9 \pm 8.5 , range 80-110).

The average DQ was significantly lower $(P<0.0005)$ in the 57 subjects with athyreosis (mean 94.6 \pm 12.2 , range 80-122) than in those with other types of $\mathrm{CH}$ (mean 102.2 \pm 10.3 , range 80-130).

In the entire study population the overall prevalence of cases with abnormal DQ was 15/192 (3.7\%). This prevalence was significantly lower in group A than in group B $\left(4 / 107\right.$ vs $\left.11 / 85 ; \chi^{2}=5.57, P<0.025\right)$, whereas no differences were found between subgroups $\mathrm{B} 1$ and B2 (5/40 vs $6 / 45)$.

An abnormal DQ was found more frequently in athyreotic patients than in the patients with other types of $\mathrm{CH}\left(10 / 57\right.$ vs $\left.5 / 135 ; \chi^{2}=10.65, P<0.005\right)$.

No significant relationships were found between DQ and pre-treatment thyroid function tests, both at the time of screening and at recall. Moreover, DQ was associated with none of the variables related to therapy (age at therapy onset, initial L- $\mathrm{T}_{4}$ dose, age at TSH normalization, average TSH and $\mathrm{T}_{4}$ serum levels during the first year of life under $\mathrm{L}-\mathrm{T}_{4}$ therapy).

\section{Discussion}

An important fact which emerges from the present investigation is that in a cohort of 192 infants with $\mathrm{CH}$ all detected by the neonatal screening, a number of patients still show retardation in psychomotor development, despite immediate and adequate substitutive treatment. In the last few years several studies have investigated psychomotor development and intellectual outcome of subjects with $\mathrm{CH}$ diagnosed by neonatal screening. Some of them found no differences in cognitive performances between $\mathrm{CH}$ patients and controls $(18,19)$, whilst other studies demonstrated the existence of some minor deficits in $\mathrm{CH}$ individuals $(1-11)$.

Subsequent clinical studies have shown that a number of variables influence DQ in children with $\mathrm{CH}$. These include: timing of treatment, initial dose of $\mathrm{L}-\mathrm{T}_{4}$, severity of $\mathrm{CH}$ (very low serum $\mathrm{T}_{4}$ concentrations before therapy onset, athyreosis, delayed skeletal maturation at birth) and average serum $\mathrm{FT}_{4}$ levels during the first year of life.

Age at the onset of replacement therapy in $\mathrm{CH}$ had already been demonstrated to be a very important determinant of intellectual outcome in the pre-screening era (20) and screening programs aim to reduce the postnatal duration of the hypothyroid period as much as possible. According to a recent study, a delayed start of treatment might lower intellectual outcome several points per week during the early weeks of extrauterine life (21). Nevertheless, according to other recent reports, a small delay of substitutive treatment in infants diagnosed by newborn screening does not seem to be an important risk factor for a poor intellectual outcome (22-24).

Another important determinant of psychomotor development in $\mathrm{CH}$ infants diagnosed by newborn screening is the initial dose of $\mathrm{L}-\mathrm{T}_{4}$ therapy, and there is a growing consensus that initial doses should be higher than those which were generally used at the start of $\mathrm{CH}$ screening programs $(25,26)$. This view has recently been supported by the finding of a significant association between $\mathrm{L}_{\mathrm{L}} \mathrm{T}_{4}$ dosage at the onset of treatment and subsequent school performance achievement (24).

CH severity parameters are well documented determinants of subsequent neurocognitive deficits in subjects with $\mathrm{CH}$ (24). In some studies $\mathrm{CH}$ severity has been found to be significantly associated with neurocognitive outcome, probably as a consequence of the deleterious effects of prenatal thyroid failure on central nervous system maturation $(4,27)$. However, this negative impact of severe versus mild $\mathrm{CH}$ on $\mathrm{DQ}$ can be counteracted by an early and high dose $\mathrm{L}_{\mathrm{T}} \mathrm{T}_{4}$ treatment $(19,28)$. On the other hand, children with mild $\mathrm{CH}$ who are usually prone to a normal psychomotor development, may also experience an insufficient long term evolution if treated late with a low initial $\mathrm{L}_{-} \mathrm{T}_{4}$ dose (21). The results of these studies suggest that an optimal treatment can lead to a very good outcome in individuals with severe $\mathrm{CH}$, whereas even children with mild $\mathrm{CH}$ are vulnerable in cases of non-optimal treatment (2).

The importance of an optimal treatment - based on an early start of high doses of $\mathrm{L}-\mathrm{T}_{4}$ and careful followup of the adequacy of therapy - has recently been confirmed by Léger et al. (24). These authors demonstrated for the first time that the adequacy of treatment during follow-up is the strongest factor related to school performance achievement in $\mathrm{CH}$ adolescents, even when the disease severity at diagnosis is taken into account.

In many of the previous reports, the above considered treatment variables (age at therapy initiation, initial $\mathrm{L}-\mathrm{T}_{4}$ dose, severity of $\mathrm{CH}$ and adequacy of treatment) frequently overlapped, which did not allow definite conclusions to be drawn about the autonomous role played by each of these factors in conditioning psychomotor development in $\mathrm{CH}$ infants.

Ours is the first investigation based on a study design aiming to evaluate the influence on psychomotor development of only one variable ( $\mathrm{CH}$ severity), having preliminarily excluded the interference of all the treatment variables (age at therapy onset, initial $\mathrm{L}-\mathrm{T}_{4}$ dose, adequacy of treatment during the first year). This study 
design gave us the opportunity of showing that $\mathrm{CH}$ severity per se can significantly affect psychomotor development during the first year of life, even in the patients who are treated with adequate amounts of $\mathrm{L}^{-\mathrm{T}_{4}}$ within 1 month after birth and who attain early normalization of TSH serum levels (before the age of 3 months). In our series, psychomotor outcome at the age of 1 year was significantly conditioned by $\mathrm{CH}$ severity parameters irrespective of treatment variables. The lowest scores in terms of DQ were observed in the subjects with delayed BM at birth and particularly in those with athyreosis while other pre-treatment factors, such as thyroid function tests, were not significantly able to predict DQ at the age of 1 year.

In accordance with recent reports $(24,29)$, our data indicate that high $\mathrm{L}^{-} \mathrm{T}_{4}$ starting doses rapidly normalize serum TSH concentrations resulting in a normalization of psychomotor development in almost all (96.3\%) the patients. In the few patients of our series with slightly abnormal DQ (between 80 and 85) at the age of one year their negative performances were significantly related to $\mathrm{CH}$ severity parameters. This observation seems to support the recent view that children with athyreosis need more careful evaluation and a more aggressive treatment schedule (1).

Our results suggest that a closer monitoring schedule should be adopted during the first year of life in all individuals with delayed BM at birth, not only in those with athyreosis. This implies that BM should be assessed in all $\mathrm{CH}$ patients before the initiation of therapy, a procedure that is not routine in most centers.

In the present study BM was scored for the first time in a different way according to whether distal femur bony nucleus is either absent or hypoplasic, in order to evaluate whether a different degree of BM retardation may reflect a different impairment of thyroid function and thus have a different impact on psychomotor development. Our data demonstrated that the absence of distal femur nucleus is frequently associated with a more severe hypothyroid picture. No differences, however, in terms of $\mathrm{DQ}$ were found between the patients with a different degree of BM at birth. This implies that both infants with absent bony nucleus and those with hypoplasic epiphyseal nucleus are exposed to an increased risk of developing subtle defects in psychomotor development and should, therefore, undergo a more careful clinical and biochemical monitoring during the first year of life.

That athyreosis and delay in BM are important factors influencing psychomotor development in $\mathrm{CH}$ is a fact which has been known for a long time (30). In previous studies, however, the initial treatment with $\mathrm{L}_{-} \mathrm{T}_{4}$ was not as optimal as in the present investigation and the possibility that other variables related to treatment may have played some role in conditioning the poor psychomotor outcome cannot be excluded.

To sum up, our results as a whole demonstrate that: (a) $\mathrm{BM}$ at diagnosis of $\mathrm{CH}$ is delayed in almost half of the patients; (b) the retardation of $\mathrm{BM}$ in $\mathrm{CH}$ infants reflects a more severe thyroid function impairment and is frequently associated with athyreosis; (c) $\mathrm{CH}$ severity per se can significantly correlate with psychomotor development during the first year of life, irrespective of other variables related to treatment; (d) among the subjects with BM delay at birth a different degree of BM retardation may reflect a different impairment of thyroid function, without being associated with any different impact on psychomotor development during the first year of life.

\section{References}

1 Hanukoglu A, Perlman K, Shamis I, Brnjac L, Rovet J \& Daneman D. Relationship of etiology to treatment in congenital hypothyroidism. Journal of Clinical Endocrinology and Metabolism $200186186-191$.

2 Heyerdahl S. Longterm outcome in children with congenital hypothyroidism. Acta Paediatrica $2001901220-1222$.

3 Glorieux J, Dussault JH, Morisette J, Desjardins M, Letarte J \& Guyda H. Follow-up at ages 5 and 7 years on mental development in children with hypothyroidism detected by the Quebec Screening Program. Journal of Pediatrics 1985107 913-915.

4 Tillotson SL, Fuggle PW, Smith I, Ades AE \& Grant DB. Relation between biochemical severity and intelligence in early treated congenital hypothyroidism: a threshold effect. British Medical Journal $1994309440-445$.

5 Kooistra L, Laane C, Vulsma T, Schellekens JMH, Van der Meere JJ \& Kalverboer AF. Motor and cognitive development in children with congenital hypothyroidism: a long-term evaluation of the effects of neonatal treatment. Journal of Pediatrics $1994 \mathbf{1 2 4}$ 903-909.

6 Salerno M, Di Maio S, Militerni R, Argenziano A, Valerio G \& Tenore A. Prognostic factors in the intellectual development at 7 years of age in children with congenital hypothyroidism. Journal of Endocrinological Investigation 199518 774-779.

7 Simmons WF, Fuggle PW, Grant DB \& Smith I. Educational progress, behaviour and motor skills at 10 years in early treated congenital hypothyroidism. Archives of Disease in Childhood 199777 219-222.

8 Rovet JF. Congenital hypothyroidism: long-term outcome. Thyroid $19999741-748$.

9 Rovet J, Ehrlich R \& Sorbara D. Intellectual outcome in children with fetal hypothyroidism. Journal of Pediatrics $1987 \mathbf{1 1 0}$ 700-704.

10 Glorieux J, Desjardins M, Letarte J, Morissette J \& Dussault JH. Useful parameters to predict the eventual mental outcome of hypothyroid children. Pediatric Research 198824 6-8.

11 Wolter R, Noel P, De Cock P, Ernould C, Malvaux P, Verstaeten F et al. Neuropsychological study in treated thyroid dysgenesis. Acta Paediatrica Scandinavica 1980277 (Suppl) 41-46.

12 Garcia MF, Calzada-Leon R, Perez JR, Martinez MPG, Gonzalez RP, Perez MC et al. Longitudinal assessment of $\mathrm{L}_{-} \mathrm{T}_{4}$ therapy for congenital hypothyroidism: differences between athyreosis vs ectopia and delayed vs normal bone age. Journal of Pediatric Endocrinology and Metabolism 200013 63-69.

13 Ilicki A, Larsson A \& Mortensson W. Neonatal skeletal maturation in congenital hypothyroidism and its prognostic value for psychomotor development at 3 years in patients treated early. Hormone Research $199033260-264$.

14 Sénécal J, Grose MC, Vincent A, Simon J \& Lefreche JN. Maturation osseuse de foetus et du nouveau-né. Archives Francaises de Pédiatrie $19772913-22$.

15 Heyerdahl S, Kase BF \& Stake G. Skeletal maturation during thyroxine treatment in children with congenital hypothyroidism. Acta Paediatrica 199483 618-622. 
16 Brunet O \& Lézine I. Le Development Psychologique de la Première Enfance. Paris: Presses Universitaire de France, 1951.

17 Radetti G, Gentili L, Paganini C, Oberhofer R, Deluggi I \& Deluca A. Psychomotor and audiological assessment of infants born to mothers with subclinical thyroid dysfunction in early pregnancy. Minerva Pediatrica 200052 691-698.

18 Toublanc JE, Rives S, Acosta A \& Chicaud J. Psychomotor and intellectual development in 52 children with congenital hypothyroidism screened at birth: Factors likely to have an effect on prognosis. Archives Francaises de Pédiatrie 199047 191-195.

19 Illig R, Largo RH, Weber M, Augsburger T, Lipp A, Wissler D et al. Sixty children with congenital hypothyroidism detected by neonatal thyroid: mental development at 1,4 , and 7 years: a longitudinal study. Acta Endocrinologica 1986279 346-353.

20 Klein AH, Meltzer S \& Kenny FM. Improved prognosis in congenital hypothyroidism treated before age three months. Journal of Pediatrics $197281912-915$.

21 Bongers-Schokking JJ, Koot HM, Wiersma D, Verkerk PH \& de Muinck Keizer-Shrama SMPF. Influence of timing and dose of thyroid hormone replacement on development in infants with congenital hypothyroidism. Journal of Pediatrics $2000 \mathbf{1 3 6}$ 292-297.

22 Bargagna S, Dinetti D, Pinchera A, Marcheschi M, Montanelli L, Presciuttini S et al. School attainments in children with congenital hypothyroidism detected by neonatal screening and treated early in life. European Journal of Endocrinology 1999140 407-413.

23 Rovet JF \& Ehrlich R. Psychoeducational outcome in children with early-treated congenital hypothyroidism. Pediatrics 2000 105 515-522.

24 Léger J, Larroque B \& Norton J. Influence of severity of congenital hypothyroidism and adequacy of treatment on school achievement in young adolescents: a population-based cohort study. Acta Paediatrica $2001901249-1256$.

25 Fisher DA. The importance of early management in optimizing IQ in infants with congenital hypothyroidism. Journal of Pediatrics $2000136273-274$.

26 Working Group on Neonatal Screening of the European Society for Paediatric Endocrinology, Revised guidelines for neonatal screening programmes for primary congenital hypothyroidism. Hormone Research 199952 49-52.

27 Derksen-Lubsen G \& Verkerk PH. Neuropsychologic development in early treated congenital hypothyroidism: analysis of literature data. Pediatric Research 199639 561-566.

28 Dubuis JM, Glorieux J, Richer F, Deal CL, Dussault JH \& Van Vliet G. Outcome of severe congenital hypothyroidism: closing the developmental gap with early high dose levothyroxine treatment. Journal of Clinical Endocrinology and Metabolism $1996 \mathbf{8 1}$ $222-227$.

29 Salerno M, Militerni R, Bravaccio C, Micillo M, Capalbo D, Di Maio $S$ et al. Effect of different starting doses of levothyroxine on growth and intellectual outcome at four years of age in congenital hypothyroidism. Thyroid 200212 45-52.

30 Rochiccioli P, Dutau G, Roge B, Petrus M, Augier D \& Enjaume C. Neonatal screening of hypothyroidism in France. One million quantitative analyses and psycho-motor development of 20 detected cases. Journal de Génétique Humaine 198129 13-22.

Received 27 September 2002

Accepted 8 April 2003 